\title{
Issues and futures
}

Coordinator

Geoff Worton 